\title{
Pullback Attractor for Nonautonomous Ginzburg-Landau Equation with Additive Noise
}

\author{
Yangrong Li and Hongyong Cui \\ School of Mathematics and Statistics, Southwest University, Chongqing 400715, China \\ Correspondence should be addressed to Hongyong Cui; chy0321@126.com
}

Received 23 February 2014; Accepted 29 August 2014; Published 19 October 2014

Academic Editor: Yansheng Liu

Copyright (C) 2014 Y. Li and H. Cui. This is an open access article distributed under the Creative Commons Attribution License, which permits unrestricted use, distribution, and reproduction in any medium, provided the original work is properly cited.

Long time behavior of stochastic Ginzburg-Landau equations with nonautonomous deterministic external forces, dispersion coefficients, and nonautonomous perturbations is studied. The domain is taken as a bounded interval $\mathscr{I}$ in $\mathbb{R}$. By making use of Sobolev embeddings and Gialiardo-Nirenberg inequality we obtain the existence and upper semicontinuity of the pullback attractor in $L^{2}(\mathscr{I})$ for the equation. The upper semicontinuity shows the stability of attractors under perturbations.

\section{Introduction}

Consider the following stochastic complex Ginzburg-Landau equation with nonautonomous deterministic external force and dispersion coefficients:

$$
\begin{aligned}
\mathrm{d} u= & {\left[(\lambda+i \alpha(t)) \Delta u-(\kappa+i \beta(t))|u|^{2} u+\delta u+g(x, t)\right] \mathrm{d} t } \\
& +\varepsilon h(x) \mathrm{d} W(t),
\end{aligned}
$$

where $u(x, t)$ is unknown and complex-valued; $x \in \mathscr{I}=$ ] $0,1[\subseteq \mathbb{R}$; dispersion coefficients $\alpha(t), \beta(t)$ and external force $g(x, t)$ are all time-dependent; $\lambda, \kappa, \delta$, and $\varepsilon$ are positive real constants; $h(x)$ is a given complex-valued function; $W(t)$ is supposed to be a real-valued Wiener process on a complete probability space. (For detail of conditions see Lemma 12.)

As a kind of Schrödinger type equation, complex Ginzburg-Landau (G.-L.) equations arise in various areas of physics and chemistry as pointed out by Temam [1]. Due to their rich mathematical properties, Ginzburg-Landau equations have drawn much attention of mathematicians and many related articles appear in literature, such as [1-7]. In [15], existence and attractors for deterministic G.-L. equations are studied and in $[6,7]$, the authors take perturbations into consideration and have investigated the existence of attractors of solutions.
This paper deals with a nonautonomous case, equation (GL). Note that, except for perturbations, external force $g(t)$ and dispersions $\alpha(t)$ and $\beta(t)$ are all time-dependent. To investigate the long time behavior of solutions for such stochastic equations with nonautonomous deterministic terms, Wang $[8,9]$ generalized the theory on existence and upper semicontinuity of attractors for random dynamical systems (RDS), which provides us with abstract criteria (see Lemmas 10 and 11) to investigate system (GL).

Since the spatial domain $\mathscr{I}$ is bounded and the G.L. equation has smoothing effect, Sobolev embeddings are applied to obtain the asymptotic compactness of the system, as well as constructing a compact random absorbing set, which is needed to investigate not only the existence, but also the upper semicontinuity (or the stability at $\varepsilon=0$ ) of pullback attractors for G.-L. system (GL). GialiardoNirenberg inequality also contributes to estimation of the nonlinear term. 22

The main result of this paper contains Theorems 20 and

This paper is organized as follows. In Section 2 we recall some concepts and abstract results on attractors of RDS and define a cocycle for nonautonomous system (GL). Uniform estimates of solutions are established in Section 3. The main result of this paper is concluded in the last section. 
Notations. In this paper, any function space should be considered complex-valued except that there are particular explanations. We denote by $\|\cdot\|_{X}$ the norm of any (complex- or real-valued) Banach space $X$. The norm of a $q$-times Lebesgue integrable space $L^{q}(\mathscr{I}), 0<q \leqslant \infty$, is denoted by $\|\cdot\|_{q}$ for short and $\|\cdot\|_{2}=:\|\cdot\|$. We often write $H=L^{2}(\mathscr{I})$ and $V=$ $H_{0}^{1}(\mathscr{I})$ for convenience and denote the inner product of $H$ by $(\cdot, \cdot)$ with $(u, v)=\int_{\mathscr{I}} u \bar{v} \mathrm{~d} x$, for all $u, v \in H$. To investigate the stability of attractors, we use subscript " ${ }_{\varepsilon}$ " or superscript “ $\varepsilon$ " to indicate the dependence on $\varepsilon$ when necessary. Letter $c$ stands for positive constants independent of $\varepsilon$ and may change its value from line to line; $c(\cdots)$ indicates the exclusive dependence on the parameters in the bracket.

\section{Preliminary Result and the Cocycle}

2.1. Pullback Attractors for Cocycles. In this part we recall some theory of pullback attractors for RDS with nonautonomous deterministic terms. The reader is referred to [810] for more details.

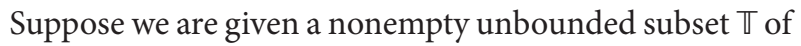
$\mathbb{R}$, a probability space $(\Omega, \mathscr{F}, \mathbb{P})$ and a Banach space $\left(X,\|\cdot\|_{X}\right)$ with Borel $\sigma$-algebra $\mathscr{B}(X)$.

Definition 1. A nonautonomous random set $B=$ $\{B(\tau, \omega)\}_{\tau, \omega}:=\{B(\tau, \omega): \tau \in \mathbb{T}, \omega \in \Omega\}$ of $X$ is a setvalued mapping: $\mathbb{T} \times \Omega \rightarrow 2^{X}$ which is measurable with respect to $\mathscr{F}$ in $\Omega$. That is, the value $B(\tau, \omega)$ of every $(\tau, \omega) \in \mathbb{T} \times \Omega$ is a closed nonempty subset of $X$ and the mapping $\omega \in \Omega \rightarrow \mathrm{d}(x, B(\tau, \omega))$ is $(\mathscr{F}, \mathscr{B}(\mathbb{R}))$-measurable for each fixed $x \in X$ and $\tau \in \mathbb{T}$.

Definition 2. Suppose there are two groups $\left\{\Theta_{s}\right\}_{s \in \mathbb{R}}$ and $\left\{\theta_{s}\right\}_{s \in \mathbb{R}}$ acting on $\mathbb{T}$ and $\Omega$, respectively. ( $\mathbb{T},\left\{\Theta_{s}\right\}_{s \in \mathbb{R}}$ ) and $\left(\Omega, \mathscr{F}, \mathbb{P},\left\{\theta_{s}\right\}_{s \in \mathbb{R}}\right)$ are called two parametric dynamical systems if

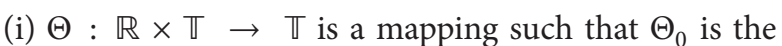
identity operator on $\mathbb{T}$;

(ii) $\Theta_{s+t}=\Theta_{t} \circ \Theta_{s}$ for all $t, s \in \mathbb{R}$;

(i') $\theta: \mathbb{R} \times \Omega \rightarrow \Omega$ is a $(\mathscr{B}(\mathbb{R}) \times \mathscr{F}, \mathscr{F})$-measurable mapping such that $\theta_{0}$ is the identity operator on $\Omega$;

(ii') $\theta_{s+t}=\theta_{s} \circ \theta_{t}$ for all $s, t \in \mathbb{R}$ and $\theta_{s} \mathbb{P}=\mathbb{P}$ for all $s \in \mathbb{R}$.

Definition 3. Class $\mathscr{D}_{\gamma}$ is defined for any fixed $\gamma \in \mathbb{R}^{+}$as the collection of all nonempty and bounded nonautonomous random sets of $X$, of which every member $B=\{B(\tau, \omega)\}_{\tau, \omega}$ satisfies

$$
\lim _{t \rightarrow+\infty} e^{-\gamma t}\left\|B\left(\Theta_{-t} \tau, \theta_{-t} \omega\right)\right\|_{X}^{2}=0
$$

for each $\tau \in \mathbb{T}$ and $\mathbb{P}$-a.e. $\omega \in \Omega$,

where $\|B\|_{X}=\sup _{x \in B}\|x\|_{X}$.

Definition 4. Let $\left(\mathbb{T},\left\{\Theta_{s}\right\}_{s \in \mathbb{R}}\right)$ and $\left(\Omega, \mathscr{F}, \mathbb{P},\left\{\theta_{s}\right\}_{s \in \mathbb{R}}\right)$ be two parametric dynamical systems. A mapping $\phi: \mathbb{R}^{+} \times \mathbb{T} \times$ $\Omega \times X \rightarrow X$ is called a cocycle on $X$ over $\left(\mathbb{T},\left\{\Theta_{s}\right\}_{s \in \mathbb{R}}\right)$ and
$\left(\Omega, \mathscr{F}, \mathbb{P},\left\{\theta_{s}\right\}_{s \in \mathbb{R}}\right)$ if for all $\tau \in \mathbb{T}, \omega \in \Omega$, and $s, t \in \mathbb{R}^{+}$, it satisfies that

(i) $\phi(\cdot, \tau, \cdot) \cdot: \mathbb{R}^{+} \times \Omega \times X \rightarrow X$ is $\left(\mathscr{B}\left(\mathbb{R}^{+}\right) \times \mathscr{F} \times\right.$ $\mathscr{B}(X), \mathscr{B}(X))$-measurable;

(ii) $\phi(0, \tau, \omega)$ is the identity operator on $X$;

(iii) $\phi(t+s, \tau, \omega)=\phi\left(t, \Theta_{s} \tau, \theta_{s} \omega\right) \circ \phi(s, \tau, \omega)$.

If, in addition, $\phi(t, \tau, \omega): X \rightarrow X$ is always a continuous mapping on $X, \phi$ is called a continuous cocycle.

Let in the sequel $\phi$ be a continuous cocycle on a Banach space $X$ over $\left(\mathbb{T},\left\{\Theta_{s}\right\}_{s \in \mathbb{R}}\right)$ and $\left(\Omega, \mathscr{F}, \mathbb{P},\left\{\theta_{s}\right\}_{s \in \mathbb{R}}\right)$.

Definition 5. Suppose $E=\{E(\tau, \omega)\}_{\tau, \omega} \in \mathscr{D}_{\gamma}$. Then $E$ is called a $\mathscr{D}_{\gamma}$-pullback absorbing set for $\phi$ if for all $\tau \in \mathbb{T}$, $\mathbb{P}$-a.e. $\omega \in \Omega$ and, for every $B \in \mathscr{D}_{\gamma}$, there exists a $T=T(B, \tau, \omega)>0$ such that

$$
\phi\left(t, \Theta_{-t} \tau, \theta_{-t} \omega\right) B\left(\Theta_{-t} \tau, \theta_{-t} \omega\right) \subseteq E(\tau, \omega) \quad \forall t \geqslant T .
$$

If, in addition, for each $\tau \in \mathbb{T}$ and $\mathbb{P}$-a.e. $\omega \in \Omega, E(\tau, \omega)$ is a closed subset of $X$ and $E$ is measurable with respect to $\mathscr{F}$ in $\Omega$, then we say $E$ is a closed measurable $\mathscr{D}_{\gamma}$-pullback absorbing set for $\phi$.

Definition 6. $\phi$ is said to be $\mathscr{D}_{\gamma}$-pullback asymptotically compact in $X$ if for all $\tau \in \mathbb{T}$ and $\mathbb{P}$-a.e. $\omega \in \Omega$, sequence $\left\{\phi\left(t_{n}, \Theta_{-t_{n}} \tau, \theta_{-t_{n}} \omega, x_{n}\right)\right\}_{n=1}^{\infty}$ has a convergent subsequence in $X$ whenever $x_{n} \in B\left(\Theta_{-t_{n}} \tau, \theta_{-t_{n}} \omega\right)$ with $\{B(\tau, \omega)\}_{\tau, \omega} \in \mathscr{D}_{\gamma}$ and $t_{n} \rightarrow \infty$.

Definition 7. A random variable $R(\omega): \Omega \rightarrow(0, \infty)$ is called tempered with respect to $\left\{\theta_{t}\right\}_{t \in \mathbb{R}}$ if

$$
\lim _{t \rightarrow \pm \infty} \frac{1}{t} \log R\left(\theta_{t} \omega\right)=0 \quad \mathbb{P} \text {-a.s., }
$$

where $t \rightarrow-\infty$ applies only to two-sided time.

Proposition 8. By Definition 7 the following properties hold true.

(i) If a random variable $R(\omega)$ is tempered, then

$$
\begin{aligned}
\lim _{t \rightarrow+\infty} e^{-\epsilon t} R\left(\theta_{-t} \omega\right)=0, \quad \lim _{t \rightarrow+\infty} e^{-\epsilon t} R\left(\theta_{t} \omega\right) & =0 \\
\forall \epsilon & >0 .
\end{aligned}
$$

(ii) If random variables $R_{1}(\omega)$ and $R_{2}(\omega)$ are both tempered, so are $R_{1}(\omega)+R_{2}(\omega)$ and $R_{1}(\omega) R_{2}(\omega)$.

Definition 9. A nonautonomous random set $\mathscr{A}=\{A(\tau, \omega)\}_{\tau, \omega}$ of $X$ is called a $\mathscr{D}_{\gamma}$-pullback attractor for $\phi$ if the following conditions are satisfied for every $\tau \in \mathbb{T}$ and $\mathbb{P}$-a.e. $\omega \in \Omega$. Consider

(i) $\mathscr{A} \in \mathscr{D}_{\gamma}$ and $A(\tau, \omega)$ is compact in $X$;

(ii) $\mathscr{A}$ is invariant; that is,

$$
\phi(t, \tau, \omega) A(\tau, \omega)=A\left(\Theta_{t} \tau, \theta_{t} \omega\right), \quad \forall t \geqslant 0 ;
$$


(iii) $\mathscr{A}$ attracts every set in $\mathscr{D}_{\gamma}$; that is, for every $B=$ $\{B(\tau, \omega)\}_{\tau, \omega} \in \mathscr{D}_{\gamma}$,

$$
\lim _{t \rightarrow \infty} \operatorname{dist}_{X}\left(\phi\left(t, \Theta_{-t} \tau, \theta_{-t} \omega\right) B\left(\Theta_{-t} \tau, \theta_{-t} \omega\right), A(\tau, \omega)\right)=0
$$

where $\operatorname{dist}_{X}(\cdot, \cdot)$ denotes the Hausdorff semimetric in $X$ defined as

$$
\operatorname{dist}_{X}(Y, Z)=\sup _{y \in Y} \inf _{z \in Z}\|y-z\|_{X}, \quad \forall Y, Z \subseteq X
$$

The following results are borrowed from Wang [8-11].

Lemma 10. Let $\phi$ be a continuous cocycle on $X$ over $\left(\Omega, \mathscr{F}, \mathbb{P},\left\{\theta_{s}\right\}_{s \in \mathbb{R}}\right)$ and $\left(\mathbb{T},\left\{\Theta_{s}\right\}_{s \in \mathbb{R}}\right)$. Then $\phi$ has a unique $\mathscr{D}_{\gamma^{-}}$ pullback attractor $\mathscr{A}$ in $\mathscr{D}_{\gamma}$ if and only if $\phi$ is $\mathscr{D}_{\gamma}$-pullback asymptotically compact in $X$ and $\phi$ has a closed measurable $\mathscr{D}_{\gamma}$-pullback absorbing set $E$ in $\mathscr{D}_{\gamma}$.

Given a metric space $\Lambda$. For every $\lambda \in \Lambda$, let $\phi_{\lambda}$ be a continuous cocycle over $\left(\mathbb{T},\left\{\Theta_{s}\right\}_{s \in \mathbb{R}}\right)$ and $\left(\Omega, \mathscr{F}, \mathbb{P},\left\{\theta_{s}\right\}_{s \in \mathbb{R}}\right)$.

Lemma 11. Suppose for each $\lambda \in \Lambda$, $\phi_{\lambda}$ has a $\mathscr{D}_{\gamma}$-pullback attractor $\mathscr{A}_{\lambda}=\left\{A_{\lambda}(\tau, \omega)\right\}_{\tau, \omega}$, particularly, $\mathscr{A}_{\lambda_{0}}=\left\{A_{\lambda_{0}}(\tau)\right\}_{\tau}$; then for every $\tau \in \mathbb{R}$ and $\omega \in \Omega$,

$$
\operatorname{dist}_{X}\left(A_{\lambda}(\tau, \omega), A_{\lambda_{0}}(\tau, \omega)\right) \longrightarrow 0 \text { as } \lambda \longrightarrow \lambda_{0} \text { in } \Lambda \text {, }
$$

\section{if provided}

(i) for every $t \in \mathbb{R}^{+}, \tau \in \mathbb{R}, \omega \in \Omega$, and $\lambda_{n} \in \Lambda$ with $\lambda_{n} \rightarrow \lambda_{0}, x_{n}, x \in X$ with $x_{n} \rightarrow x$, it holds that

$$
\lim _{n \rightarrow \infty} \phi_{\lambda_{n}}(t, \tau, \omega) x_{n}=\phi_{\lambda_{0}}(t, \tau, \omega) x
$$

(ii) there exists a mapping $R_{0}(\cdot, \cdot): \mathbb{R} \times \Omega \rightarrow \mathbb{R}$ such that the family

$B=\left\{B(\tau, \omega)=\left\{x \in X:\|x\|_{X} \leqslant R_{0}(\tau, \omega)\right\}: \tau \in \mathbb{R}, \omega \in \Omega\right\}$ belongs to $\mathscr{D}_{\gamma}$;

(iii) $\lambda \in \Lambda$, $\phi_{\lambda}$ has a $\mathscr{D}_{\gamma}$-pullback absorbing set $E_{\lambda}=$ $\left\{E_{\lambda}(\tau, \omega)\right\}_{\tau, \omega}$ such that for all $\tau \in \mathbb{R}$ and $\omega \in \Omega$,

$$
\limsup _{\lambda \rightarrow \lambda_{0}}\left\|E_{\lambda}(\tau, \omega)\right\|_{X} \leqslant R_{0}(\tau, \omega)
$$

where $R_{0}$ is as in (ii) and $\|S\|_{X}=\sup _{x \in S}\|x\|_{X}$, for all $S \subseteq X$;

(iv) for every $\tau \in \mathbb{R}$ and $\omega \in \Omega$,

$$
\bigcup_{\lambda \in \Lambda} A_{\lambda}(\tau, \omega) \text { is precompact in } X .
$$

2.2. Nonautonomous Stochastic G.-L. Equations. Given $\tau \in \mathbb{R}$ and $t \geqslant \tau$, consider equations defined for $x \in \mathscr{I}=] 0,1[\subseteq \mathbb{R}$,

$$
\begin{gathered}
\mathrm{d} u=\left[(\lambda+i \alpha(t)) \Delta u-(\kappa+i \beta(t))|u|^{2} u+\delta u\right. \\
+g(x, t)] \mathrm{d} t+\varepsilon h(x) \mathrm{d} W(t),
\end{gathered}
$$

with initial-boundary value conditions

$$
u(x, \tau)=u_{0}(x),\left.\quad u(x, \cdot)\right|_{\partial \mathscr{I}} \equiv 0
$$

where the unknown $u(x, t)$ is a complex-valued function; dispersion coefficients $\alpha(t), \beta(t)$, and external force $g(x, t)$ are all time-dependent and real-valued functions; $\lambda, \kappa, \delta$, and $\varepsilon \in] 0,1]$ are positive constants; $h(x) \in H_{0}^{1}(\mathscr{I}) \cap H^{2}(\mathscr{I}) ; W(t)$ is supposed to be a two-sided real-valued Wiener process on a complete probability space $(\Omega, \mathscr{F}, \mathbb{P})$, where $\Omega=\{\omega \epsilon$ $C(\mathbb{R}, \mathbb{R}): \omega(0)=0\}, \mathscr{F}$ is Borel $\sigma$-algebra induced by the compact open topology of $\Omega$, and $\mathbb{P}$ is the corresponding Wiener measure on $(\Omega, \mathscr{F})$.

To define a proper cocycle for system (13), we first define the related parametric dynamic systems as follows. Let $\left\{\Theta_{s}\right\}_{s \in \mathbb{R}}$ be the group acting on $\mathbb{R}$ satisfying

$$
\Theta_{s} \tau=\tau+s \quad \forall s, \tau \in \mathbb{R},
$$

and $\left\{\theta_{s}\right\}_{s \in \mathbb{R}}$ the group acting on $(\Omega, \mathscr{F}, \mathbb{P})$ such that

$$
\theta_{s} \omega(\cdot)=\omega(\cdot+s)-\omega(s) \quad \forall \omega \in \Omega, s \in \mathbb{R},
$$

where we have identified $W(t)$ with

$$
W(t, \omega)=\omega(t), \quad t \geqslant \tau .
$$

Then by Definition 2 it is evident that $\left(\mathbb{R},\left\{\Theta_{s}\right\}_{s \in \mathbb{R}}\right)$ and $\left(\Omega, \mathscr{F}, \mathbb{P},\left(\theta_{s}\right)_{s \in \mathbb{R}}\right)$ are two parametric dynamical systems.

We now define a continuous cocycle $\phi$ over $\left(\mathbb{R},\left\{\Theta_{s}\right\}_{s \in \mathbb{R}}\right)$ and $\left(\Omega, \mathscr{F}, \mathbb{P},\left\{\theta_{s}\right\}_{s \in \mathbb{R}}\right)$ for system (13). Consider the onedimensional Ornstein-Uhlenbeck equation

$$
\mathrm{d} y-\delta y \mathrm{~d} t=\mathrm{d} W,
$$

of which a stationary solution is provided by

$$
y(t)=y\left(\theta_{t} \omega\right) \equiv-\delta \int_{0}^{\infty} e^{-\delta \tau}\left(\theta_{t} \omega\right)(\tau) \mathrm{d} \tau, \quad t \in \mathbb{R} .
$$

It is known that there exists a $\theta_{t}$-invariant set $\widetilde{\Omega} \subseteq \Omega$ with $\mathbb{P}(\widetilde{\Omega})=1$ such that $y\left(\theta_{t} \omega\right)$ is continuous in $t$ for every $\omega \epsilon$ $\widetilde{\Omega}$, and the random variable $\left|y\left(\theta_{t} \omega\right)\right|$ is tempered (see, e.g., $[8,10,12,13]$, and hereafter we will not distinguish $\widetilde{\Omega}$ from $\Omega$ ). Therefore, by Proposition 8 and [12, Proposition 4.3.3] (see also [14-16]), there exists a tempered variable $r(\omega)>0$ such that

$$
|y(\omega)|^{6} \leqslant r(\omega)
$$

where $r(\omega)$ satisfies

$$
r\left(\theta_{t} \omega\right) \leqslant e^{(\gamma / 2)|t|} r(\omega), \quad t \in \mathbb{R} .
$$


Let $z\left(\theta_{t} \omega\right)=h y\left(\theta_{t} \omega\right), f(s)=|s|^{2} s$, and

$$
\begin{array}{r}
v\left(t, \tau, \omega, v_{0}\right)=u\left(t, \tau, \omega, u_{0}\right)-\varepsilon z\left(\theta_{t} \omega\right) \\
\text { with } v_{0}=u_{0}-\varepsilon z\left(\theta_{\tau} \omega\right) .
\end{array}
$$

Then if $u(t)$ solves (13)-(14), $v(t)$ should satisfy, by (18), (22), and $h(x) \in H_{0}^{1}(\mathscr{I}) \cap H^{2}(\mathscr{I})$,

$$
\begin{aligned}
\frac{\mathrm{d} v}{\mathrm{~d} t}= & (\lambda+i \alpha(t)) \Delta v-(\kappa+i \beta(t)) f\left(v+\varepsilon z\left(\theta_{t} \omega\right)\right) \\
& +\delta v+g(x, t)+\varepsilon(\lambda+i \alpha(t)) \Delta z\left(\theta_{t} \omega\right),
\end{aligned}
$$

with conditions

$$
\begin{gathered}
v\left(\tau, \tau, \omega, v_{0}\right)=v_{0}=u_{0}-\varepsilon z\left(\theta_{\tau} \omega\right), \\
\left.v\left(t, \tau, \omega, v_{0}\right)\right|_{\partial \mathscr{I}}=0,
\end{gathered}
$$

for all $t \geqslant \tau \in \mathbb{R}$ and $x \in \mathscr{I}=] 0,1$ [. Since (23)-(24) is a deterministic problem, by the "standard" Galërkin method as in [17] or similar arguments of [2] (see also [1, 5, 6] for autonomous G.-L. equations), we have the following wellpossessedness result.

Lemma 12. Assume that

(i) $\left.\left.\lambda \in \mathbb{R}^{+}, \kappa \in \mathbb{R}^{+}, \delta \in \mathbb{R}^{+}, \varepsilon \in\right] 0,1\right], h(x) \in H_{0}^{1}(\mathscr{I} ; \mathbb{C}) \cap$ $H^{2}(\mathscr{I} ; \mathbb{C})$;

(ii) $\beta(t) \in C_{\text {loc }}(\mathbb{R} ; \mathbb{R})$ and $\sup _{t \in \mathbb{R}}|\beta(t)| \leqslant \sqrt{3} \kappa$;

(iii) $\alpha(t) \in C_{l o c}(\mathbb{R} ; \mathbb{R})$ and $g(x, t) \in L_{\text {loc }}^{2}(\mathbb{R} ; H)$ are such that

$$
\int_{-\infty}^{\tau} e^{(\gamma / 2) s}\left(|\alpha(t)|^{4}+\|g(x, s)\|^{2}\right) \mathrm{d} s<+\infty
$$

$$
\text { for every } \tau \in \mathbb{R} \text {. }
$$

Then, for each $v_{0} \in H$, the initial-boundary value problem (23)-(24) has a unique weak solution

$$
\begin{gathered}
v\left(t, \tau, \omega, v_{0}\right) \in C_{l o c}(\tau, \infty ; H) \cap L_{l o c}^{2}(\tau, \infty ; V) \\
\cap L_{l o c}^{4}\left(\tau, \infty ; L^{4}(\mathscr{I})\right) .
\end{gathered}
$$

Besides, $v\left(t, \tau, \omega, v_{0}\right)$ is $(\mathscr{F}, \mathscr{B}(H))$-measurable in $\omega \in \Omega$ and continuous in $v_{0}$ with respect to the norm of $H$ for each $t \geqslant \tau$.

Let

$$
\begin{array}{r}
u\left(t, \tau, \omega, u_{0}\right)=v\left(t, \tau, \omega, v_{0}\right)+\varepsilon z\left(\theta_{t} \omega\right) \\
\text { with } u_{0}=v_{0}+\varepsilon z\left(\theta_{\tau} \omega\right) .
\end{array}
$$

Then under assumptions of Lemma 12 it is evident that $u$ solves problem (13)-(14) and is $(\mathscr{F}, \mathscr{B}(H))$-measurable in $\omega \epsilon$ $\Omega$, continuous in both $t \geqslant \tau$, and $u_{0} \in H$. Consider the mapping $\phi: \mathbb{R}^{+} \times \mathbb{R} \times \Omega \times H \rightarrow H$ with

$$
\begin{aligned}
\phi\left(t, \tau, \omega, u_{0}\right) & =u\left(t+\tau, \tau, \theta_{-\tau} \omega, u_{0}\right) \\
& =v\left(t+\tau, \tau, \theta_{-\tau} \omega, v_{0}\right)+\varepsilon z\left(\theta_{t} \omega\right),
\end{aligned}
$$

where $v_{0}=u_{0}-\varepsilon z(\omega)$. By the property of solution trajectories of well-possessed nonautonomous dynamical systems one can readily check according to Definition 4 that (28) defines a continuous cocycle $\phi$ for problem (13)-(14) on $H$ over $\left(\mathbb{R},\left\{\Theta_{s}\right\}\right)$ and $\left(\Omega, \mathscr{F}, \mathbb{P},\left\{\theta_{s}\right\}\right)$, where $\left\{\Theta_{s}\right\}_{s \in \mathbb{R}}$ and $\left\{\theta_{s}\right\}_{s \in \mathbb{R}}$ are given by (15) and (16), respectively.

To investigate the $\left(\mathscr{D}_{\gamma^{-}}\right)$pullback attractor in $H$ for system (13), hereafter in this paper, we let, for an arbitrarily fixed $\gamma>0, \mathscr{D}_{\gamma}$ be the class satisfying Definition 3 with $X=H$.

\section{Uniform Estimates of Solutions}

In this section we estimate the solution of problem (13)-(14) to establish a $\mathscr{D}_{\gamma}$-random absorbing set, as well as to obtain the $\mathscr{D}_{\gamma}$-pullback asymptotic compactness for the cocycle $\phi$ under assumptions of Lemma 12. We begin with two useful lemmas.

Lemma 13 (Young's inequality). Let $a, b>0$. Then for every $p$, q satisfying $1<p, q<\infty,(1 / p)+(1 / q)=1$, it holds that

$$
a \cdot b \leqslant \epsilon \frac{a^{p}}{p}+\epsilon^{-q / p} \frac{b^{q}}{q} \quad \forall \epsilon>0 .
$$

Lemma 14 (Gagliardo-Nirenberg's inequality). Let $u \in$ $L^{q}\left(\mathbb{R}^{n}\right), D^{m} u \in L^{r}\left(\mathbb{R}^{n}\right), 1 \leqslant q, r \leqslant \infty$. Then for $0 \leqslant j<m$, $(j / m) \leqslant a<1$, there exists a constant $C$ such that

$$
\left\|D^{j} u\right\|_{p} \leqslant C\left\|D^{m} u\right\|_{r}^{a} \cdot\|u\|_{q}^{1-a}
$$

where $1 / p=(j / n)+a((1 / r)-(m / n))+(1-a)(1 / q)$.

By Lemma 14 we derive the following estimates for later convenience.

Corollary 15. Let $x \in \mathscr{I} \subseteq \mathbb{R}$. Then it holds true for every well-defined $n(x)$ that

$$
\begin{gathered}
\|n\|_{4}^{4} \leqslant C\|n\|^{3}\|\nabla n\|, \quad\|n\|_{6}^{6} \leqslant C\|n\|^{4}\|\nabla n\|^{2}, \\
\|\nabla n\|^{2} \leqslant C\|\Delta n\|^{6 / 7}\|n\|_{4}^{8 / 7} .
\end{gathered}
$$

Lemma 16. Let assumptions of Lemma 12 hold. Then for every $\tau \in \mathbb{R}, \omega \in \Omega$, and $D \in \mathscr{D}_{\gamma}$, there exists a $T=$ $T(\tau, \omega, D) \geqslant 1$ and a positive constant $L$, which depends on $\gamma$ but is independent of $\varepsilon, \tau, \omega$, and $D$, such that the solution $v\left(t, \tau, \omega, v_{0}\right)$ with $v_{0} \in D\left(\tau-t, \theta_{-\tau} \omega\right)$ of (23)-(24) satisfies, for all $t \geqslant T$, that

$$
\begin{aligned}
& \left\|v\left(\tau, \tau-t, \theta_{-t} \omega, v_{0}\right)\right\|^{2} \\
& \quad \leqslant L e^{-\gamma \tau} \int_{-\infty}^{\tau} e^{\gamma s}\left(|\alpha(t)|^{4}+\|g(x, s)\|^{2}\right) \mathrm{d} s+\varepsilon L r(\omega)+L,
\end{aligned}
$$




$$
\begin{aligned}
& \int_{\tau-t}^{\tau} e^{\gamma s}\left\|\nabla v\left(s, \tau-t, \theta_{-\tau} \omega, v_{0}\right)\right\|^{2} \mathrm{~d} s \\
& \quad+\int_{\tau-t}^{\tau} e^{\gamma s}\left\|v\left(s, \tau-t, \theta_{-\tau} \omega, v_{0}\right)\right\|_{4}^{4} \mathrm{~d} s \\
& \leqslant L \int_{-\infty}^{\tau} e^{\gamma s}\left(|\alpha(t)|^{4}+\|g(x, s)\|^{2}\right) \mathrm{d} s+\varepsilon L e^{\gamma \tau} r(\omega)+L
\end{aligned}
$$

where $r(\omega)$ is the tempered random variable given by (20) and (21).

Proof. Taking the inner product of (23) with $v$ in $H$ and taking the real part, we get

$$
\begin{aligned}
\frac{1}{2} \frac{\mathrm{d}}{\mathrm{d} t}\|v\|^{2} & \\
= & -\lambda\|\nabla v\|^{2}-\operatorname{Re}\left((\kappa+i \beta(t)) f\left(v+\varepsilon z\left(\theta_{t} \omega\right)\right), v\right)+\delta\|v\|^{2} \\
& +\varepsilon \operatorname{Re}(g(t), v)+\operatorname{Re}\left((\lambda+i \alpha) \Delta z\left(\theta_{t} \omega\right), v\right) .
\end{aligned}
$$

By conditions $f(s)=|s|^{2} s,|\beta(t)| \leqslant \sqrt{3} \kappa$, and $z\left(\theta_{t} \omega\right)=$ $h y\left(\theta_{t} \omega\right)$ we derive that

$$
\begin{aligned}
- & \operatorname{Re}\left((\kappa+i \beta(t)) f\left(v+\varepsilon z\left(\theta_{t} \omega\right)\right), v\right) \\
= & -\operatorname{Re}\left((\kappa+i \beta(t))\left|v+\varepsilon z\left(\theta_{t} \omega\right)\right|^{2}\left(v+\varepsilon z\left(\theta_{t} \omega\right)\right), v\right) \\
= & -\operatorname{Re}\left(( \kappa + i \beta ( t ) ) \left(|v|^{2} v+2 v|\varepsilon z|^{2}+\varepsilon v^{2} \bar{z}\right.\right. \\
& \left.\left.+|\varepsilon z|^{2} z+\varepsilon^{2} \bar{v} z^{2}+2 \varepsilon|v|^{2} z\right), v\right) \\
\leqslant & -\kappa\|v\|_{4}^{4} \quad \\
& +\varepsilon\left|\int_{\mathscr{I}}(\kappa+i \beta(t))\left(v|v|^{2} \bar{z}+|z|^{2} z \bar{v}+\bar{v}^{2} z^{2}+2|v|^{2} z \bar{v}\right) \mathrm{d} x\right| \\
\leqslant & -\kappa\|v\|_{4}^{4}+2 \varepsilon \kappa \int_{\mathscr{I}}\left(3|v|^{3}|z|+|z|^{3}|v|+|v|^{2}|z|^{2}\right) \mathrm{d} x \\
\leqslant & -\kappa\|v\|_{4}^{4}+\frac{\kappa}{2}\|v\|_{4}^{4}+\varepsilon c\|z\|_{4}^{4} \quad(\text { by Lemma } 13) \\
\leqslant & -\frac{\kappa}{2}\|v\|_{4}^{4}+\varepsilon c\left|y\left(\theta_{t} \omega\right)\right|^{4} \quad\left(\text { by }(31) \text { and } h \in H_{0}^{1}(\mathscr{I})\right)
\end{aligned}
$$

where $c=c(\kappa,\|h\|,\|\nabla h\|)$. Since similarly we have

$$
\begin{aligned}
& \operatorname{Re}(g(t), v)+\varepsilon \operatorname{Re}\left((\lambda+i \alpha(t)) \Delta z\left(\theta_{t} \omega\right), v\right) \\
& \leqslant \frac{1}{2}\|g(x, t)\|^{2}+\frac{1}{2}\|v\|^{2}+\frac{\lambda}{2}\|\nabla v\|^{2} \\
&+\varepsilon c|\lambda+i \alpha(t)|^{2}\left|y\left(\theta_{t} \omega\right)\right|^{2} \\
& \leqslant \frac{1}{2}\|g(x, t)\|^{2}+\frac{1}{2}\|v\|^{2}+\frac{\lambda}{2}\|\nabla v\|^{2} \\
&+\varepsilon c\left(|\alpha(t)|^{4}+\left|y\left(\theta_{t} \omega\right)\right|^{4}+\left|y\left(\theta_{t} \omega\right)\right|^{2}\right),
\end{aligned}
$$

where $c=c(\lambda,\|\nabla h\|)$; from (34)-(36) it follows that

$$
\begin{aligned}
& \frac{\mathrm{d}}{\mathrm{d} t}\|v\|^{2}+\lambda\|\nabla v\|^{2}+\kappa\|v\|_{4}^{4} \\
& \leqslant(2 \delta+1)\|v\|^{2}+\|g(x, t)\|^{2} \\
&+\varepsilon c\left(|\alpha(t)|^{4}+\left|y\left(\theta_{t} \omega\right)\right|^{4}+\left|y\left(\theta_{t} \omega\right)\right|^{2}\right),
\end{aligned}
$$

which implies that

$$
\begin{aligned}
\frac{\mathrm{d}}{\mathrm{d} t}\|v\|^{2} & +\gamma\|v\|^{2}+\lambda\|\nabla v\|^{2}+\frac{\kappa}{2}\|v\|_{4}^{4} \\
\leqslant & -\frac{\kappa}{2} \int_{\mathscr{I}}\left(|v|^{2}-\frac{2 \delta+1+\gamma}{\kappa}\right)^{2} \mathrm{~d} x \\
& +\frac{\kappa}{2} \int_{\mathscr{I}}\left(\frac{2 \delta+1+\gamma}{\kappa}\right)^{2} \mathrm{~d} x \\
& +\|g(x, t)\|^{2}+\varepsilon c\left(|\alpha(t)|^{4}+\left|y\left(\theta_{t} \omega\right)\right|^{4}+\left|y\left(\theta_{t} \omega\right)\right|^{2}\right) \\
\leqslant & \|g(x, t)\|^{2}+c|\alpha(t)|^{4}+\varepsilon c\left|y\left(\theta_{t} \omega\right)\right|^{4}+\varepsilon c,
\end{aligned}
$$

where $c=c(\gamma, \kappa, \delta)$. Multiply (38) by $e^{\gamma t}$ and integrate over ]$\tau-t, \tau\left[, t \in \mathbb{R}^{+}\right.$, to get, for each $\omega \in \Omega$,

$$
\begin{aligned}
& \left\|v\left(\tau, \tau-t, \omega, v_{0}\right)\right\|^{2}+\lambda \int_{\tau-t}^{\tau} e^{\gamma(s-\tau)}\left\|\nabla v\left(s, \tau-t, \omega, v_{0}\right)\right\|^{2} \mathrm{~d} s \\
& \quad+\frac{\kappa}{2} \int_{\tau-t}^{\tau} e^{\gamma(s-\tau)}\left\|v\left(s, \tau-t, \omega, v_{0}\right)\right\|_{4}^{4} \mathrm{~d} s \\
& \leqslant \int_{\tau-t}^{\tau} e^{\gamma(s-\tau)}\left(c|\alpha(s)|^{4}+\|g(x, s)\|^{2}\right) \mathrm{d} s \\
& \quad+\varepsilon c \int_{\tau-t}^{\tau} e^{\gamma(s-\tau)}\left(\left|y\left(\theta_{s} \omega\right)\right|^{4}+1\right) \mathrm{d} s+e^{-\gamma t}\left\|v_{0}\right\|^{2} .
\end{aligned}
$$

Notice that $|y|^{4} \leqslant|y|^{6}+c$. Therefore, replacing $\omega$ in (39) with $\theta_{-\tau} \omega$ and by (20)-(21) we obtain

$$
\begin{aligned}
&\left\|v\left(\tau, \tau-t, \theta_{-\tau} \omega, v_{0}\right)\right\|^{2} \\
&+\lambda e^{-\gamma \tau} \int_{\tau-t}^{\tau} e^{\gamma s}\left\|\nabla v\left(s, \tau-t, \theta_{-\tau} \omega, v_{0}\right)\right\|^{2} \mathrm{~d} s \\
& \quad+\frac{\kappa}{2} e^{-\gamma \tau} \int_{\tau-t}^{\tau} e^{\gamma s}\left\|v\left(s, \tau-t, \theta_{-\tau} \omega, v_{0}\right)\right\|_{4}^{4} \mathrm{~d} s \\
& \leqslant \int_{\tau-t}^{\tau} e^{\gamma(s-\tau)}\left(c|\alpha(s)|^{4}+\|g(x, s)\|^{2}\right) \mathrm{d} s \\
& \quad+\varepsilon c \int_{\tau-t}^{\tau} e^{\gamma(s-\tau)}\left(\left|y\left(\theta_{s-\tau} \omega\right)\right|^{6}+1\right) \mathrm{d} s+e^{-\gamma t}\left\|v_{0}\right\|^{2} \\
& \leqslant c e^{-\gamma \tau} \int_{-\infty}^{\tau} e^{\gamma s}\left(|\alpha(s)|^{4}+\|g(x, s)\|^{2}\right) \mathrm{d} s \\
&+\varepsilon c \int_{-\infty}^{0} e^{\gamma s} r\left(\theta_{s} \omega\right) \mathrm{d} s+c+e^{-\gamma t}\left\|D\left(\tau-t, \theta_{-t} \omega\right)\right\|^{2},
\end{aligned}
$$


where $c$ is a positive constant depending on $\epsilon$ but independent of $\varepsilon, \tau, \omega$, and $D$. Since $D \in \mathscr{D}_{\gamma}$, by Definition 3 there exists a $T=T(\tau, \omega, D) \geqslant 1$ such that

$$
e^{-\gamma t}\left\|D\left(\tau-t, \theta_{-t} \omega\right)\right\|^{2} \leqslant 1 \quad \forall t \geqslant T,
$$

which along with (40) and (20)-(21) completes the proof.

Lemma 17. Let assumptions of Lemma 12 hold. Then for every $\tau \in \mathbb{R}, \omega \in \Omega$, and $D \in \mathscr{D}_{\gamma}$, there exists a $T=$ $T(\tau, \omega, D) \geqslant 1$ and a positive constant $C$, which depends on $\gamma$ but is independent of $\varepsilon, \tau, \omega$, and $D$, such that the solution $v\left(t, \tau, \omega, v_{0}\right)$ with $v_{0} \in D\left(\tau-t, \theta_{-\tau} \omega\right)$ of (23)-(24) satisfies, for all $t \geqslant T$,

$$
\begin{aligned}
\int_{\tau-1}^{\tau} & \left\|\nabla v\left(s, \tau-t, \theta_{-\tau} \omega, v_{0}\right)\right\|^{2} \mathrm{~d} s \\
& +\int_{\tau-1}^{\tau}\left\|v\left(s, \tau-t, \theta_{-\tau} \omega, v_{0}\right)\right\|_{4}^{4} \mathrm{~d} s \\
\leqslant & C e^{-\gamma \tau} \int_{-\infty}^{\tau} e^{\gamma s}\left(|\alpha(s)|^{4}+\|g(x, s)\|^{2}\right) \mathrm{d} s+\varepsilon C r(\omega)+C,
\end{aligned}
$$

where $r(\omega)$ is the tempered random variable given by (20) and (21).

Proof. Notice that $e^{\gamma s} \geqslant e^{\gamma(\tau-1)}$ for all $\left.s \in\right] \tau-1, \tau[$. Hence, by (33) we have

$$
\begin{aligned}
\int_{\tau-1}^{\tau} & \left\|\nabla v\left(s, \tau-t, \theta_{-\tau} \omega, v_{0}\right)\right\|^{2} \mathrm{~d} s \\
& +\int_{\tau-1}^{\tau}\left\|v\left(s, \tau-t, \theta_{-\tau} \omega, v_{0}\right)\right\|_{4}^{4} \mathrm{~d} s \\
\leqslant & e^{\gamma(1-\tau)} \int_{\tau-t}^{\tau} e^{\gamma s}\left\|\nabla v\left(s, \tau-t, \theta_{-\tau} \omega, v_{0}\right)\right\|^{2} \mathrm{~d} s \\
& +e^{\gamma(1-\tau)} \int_{\tau-t}^{\tau} e^{\gamma s}\left\|v\left(s, \tau-t, \theta_{-\tau} \omega, v_{0}\right)\right\|_{4}^{4} \mathrm{~d} s \\
\leqslant & c e^{-\gamma \tau} \int_{-\infty}^{\tau} e^{\gamma s}\left(|\alpha(s)|^{4}+\|g(x, s)\|^{2}\right) \mathrm{d} s+\varepsilon c r(\omega)+c
\end{aligned}
$$

for all $t \geqslant T \geqslant 1$, which concludes the lemma.

Lemma 18. Let assumptions of Lemma 12 hold. Then for every $\tau \in \mathbb{R}, \omega \in \Omega$, and $D \in \mathscr{D}_{\gamma}$, there exists a $T=$ $T(\tau, \omega, D) \geqslant 1$ and a positive constant $K$, which depends on $\gamma$ but is independent of $\varepsilon, \tau, \omega$, and $D$, such that the solution $v\left(t, \tau, \omega, v_{0}\right)$ with $v_{0} \in D\left(\tau-t, \theta_{-\tau} \omega\right)$ of (23)-(24) satisfies, for all $t \geqslant T$,

$$
\begin{aligned}
& \left\|\nabla v\left(\tau, \tau-t, \theta_{-\tau} \omega, v_{0}\right)\right\|^{2} \\
& \leqslant \leqslant(\varepsilon r(\omega)+1) e^{-\gamma \tau} \int_{-\infty}^{\tau} e^{\gamma s}\left(|\alpha(s)|^{4}+\|g(x, s)\|^{2}\right) \mathrm{d} s \\
& \quad+K(\varepsilon r(\omega)+1)^{2},
\end{aligned}
$$

where $r(\omega)$ is the tempered random variable given by (20) and (21).

Proof. Taking the inner product of (23) with $-\Delta v$ in $H$ and taking the real part, we have

$$
\begin{aligned}
\frac{1}{2} \frac{\mathrm{d}}{\mathrm{d} t}\|\nabla v\|^{2}+\lambda\|\Delta v\|^{2} \\
=\operatorname{Re}\left((\kappa+i \beta(t)) f\left(v+\varepsilon z\left(\theta_{t} \omega\right)\right), \Delta v\right)+\delta\|\nabla v\|^{2} \\
\quad-\operatorname{Re}(g(t), \Delta v)-\varepsilon \operatorname{Re}\left((\lambda+i \alpha(t)) \Delta z\left(\theta_{t} \omega\right), \Delta v\right) .
\end{aligned}
$$

Estimate the first term in the right hand side of (45) to get (52). Since $f(s)=|s|^{2} s$, we have

$$
\begin{aligned}
\operatorname{Re}((\kappa & \left.+i \beta(t)) f\left(v+\varepsilon z\left(\theta_{t} \omega\right)\right), \Delta v\right) \\
= & \operatorname{Re}\left((\kappa+i \beta(t))\left(|v|^{2} v, \Delta v\right)\right) \\
& +\varepsilon \operatorname{Re}\left((\kappa+i \beta(t))\left(v^{2} \bar{z}+2|v|^{2} z\right), \Delta v\right) \\
& +\operatorname{Re}\left((\kappa+i \beta(t))\left(2 \varepsilon^{2} v|z|^{2}+\varepsilon^{3}|z|^{2} z+\varepsilon^{2} \bar{v} z^{2}\right), \Delta v\right) .
\end{aligned}
$$

By the condition $|\beta(t)| \leqslant \sqrt{3} \kappa$, for all $t \in \mathbb{R}$, we have

$$
\begin{aligned}
\operatorname{Re} & \left((\kappa+i \beta(t))\left(|v|^{2} v, \Delta v\right)\right) \\
= & -\operatorname{Re}\left((\kappa+i \beta(t)) \int_{\mathscr{I}}\left(|v|^{2}|\nabla v|^{2}+v \nabla|v|^{2} \nabla \bar{v}\right) \mathrm{d} x\right) \\
= & -\kappa \int_{\mathscr{I}}|v|^{2}|\nabla v|^{2} \mathrm{~d} x-\kappa \int_{\mathscr{I}} \operatorname{Re}(v \nabla \bar{v}) \nabla|v|^{2} \mathrm{~d} x \\
& +\beta(t) \int_{\mathscr{I}} \operatorname{Im}(v \nabla \bar{v}) \nabla|v|^{2} \mathrm{~d} x \\
= & -\kappa \int_{\mathscr{I}}|v|^{2}|\nabla v|^{2} \mathrm{~d} x-\frac{\kappa}{2} \int_{\mathscr{I}}\left(\nabla|v|^{2}\right)^{2} \mathrm{~d} x \\
& -\frac{i \beta(t)}{2} \int_{\mathscr{I}}(v \nabla \bar{v}-\bar{v} \nabla v) \nabla|v|^{2} \mathrm{~d} x \\
= & -\frac{1}{4} \int_{\mathscr{I}}\left(3 \kappa\left(\nabla|v|^{2}\right)^{2}+2 i \beta(t)(v \nabla \bar{v}-\bar{v} \nabla v) \nabla|v|^{2}\right. \\
& \left.+\kappa|v \nabla \bar{v}-\bar{v} \nabla v|^{2}\right) \mathrm{d} x \\
= & -\frac{1}{4} \int_{\mathscr{I}} \eta \mathbf{M} \eta^{\top} \mathrm{d} x \leqslant 0, \quad \forall t \in \mathbb{R},
\end{aligned}
$$

where $\eta^{\top}$ denotes the conjugate transpose of matrix $\eta$ and

$$
\eta=\left[\nabla|v|^{2}, v \nabla \bar{v}-\bar{v} \nabla v\right], \quad \mathbf{M}=\left[\begin{array}{cc}
3 \kappa & -i \beta \\
i \beta & \kappa
\end{array}\right] .
$$

Recall the Agmon inequality that

$$
\|z(x)\|_{\infty} \leqslant c\|z\|^{1 / 2}\|\nabla z\|^{1 / 2}, \quad x \in \mathscr{I} \subseteq \mathbb{R} .
$$


By Lemma 13, $|\beta(t)| \leqslant \sqrt{3} \kappa$, and (49) we estimate the second term in the right hand side of (46) to obtain

$$
\begin{gathered}
\varepsilon \operatorname{Re}\left((\kappa+i \beta(t))\left(v^{2} \bar{z}+2|v|^{2} z\right), \Delta v\right) \\
\leqslant \varepsilon|\kappa+i \beta(t)| \int_{\mathscr{J}} 3|v|^{2}|z||\Delta v| \mathrm{d} x \\
\leqslant \frac{\lambda}{4}\|\Delta v\|^{2}+\varepsilon c\left\|z\left(\theta_{t} \omega\right)\right\|_{\infty}^{2}\|v\|_{4}^{4} \\
\leqslant \frac{\lambda}{4}\|\Delta v\|^{2}+\varepsilon c\left|y\left(\theta_{t} \omega\right)\right|^{2}\|v\|_{4}^{4},
\end{gathered}
$$

where $c=c(\lambda, \kappa,\|h\|,\|\nabla h\|)$. Similarly, for the last term of (46) we have

$$
\begin{aligned}
& \operatorname{Re}\left((\kappa+i \beta(t))\left(2 \varepsilon^{2} v|z|^{2}+\varepsilon^{3}|z|^{2} z+\varepsilon^{2} \bar{v} z^{2}\right), \Delta v\right) \\
& \quad \leqslant 2 \varepsilon \kappa \int_{\mathscr{I}}\left(3|v||z|^{2}|\Delta v|+|z|^{3}|\Delta v|\right) \mathrm{d} x \\
& \quad \leqslant \frac{\lambda}{4}\|\Delta v\|^{2}+\varepsilon c\left(\|z\|_{\infty}^{4}\|v\|_{4}^{4}+\|z\|_{\infty}^{4}+\left\|z\left(\theta_{t} \omega\right)\right\|^{6}\right) \\
& \quad \leqslant \frac{\lambda}{4}\|\Delta v\|^{2}+\varepsilon c\left(\left|y\left(\theta_{t} \omega\right)\right|^{4}\|v\|_{4}^{4}+\left|y\left(\theta_{t} \omega\right)\right|^{4}+\left|y\left(\theta_{t} \omega\right)\right|^{6}\right) .
\end{aligned}
$$

From (46)-(51) and Lemma 13 it follows that

$$
\begin{aligned}
& \operatorname{Re}\left((\kappa+i \beta(t)) f\left(v+\varepsilon z\left(\theta_{t} \omega\right)\right), \Delta v\right) \\
& \quad \leqslant \frac{\lambda}{2}\|\Delta v\|^{2}+\varepsilon c\left(\left|y\left(\theta_{t} \omega\right)\right|^{6}+1\right)\|v\|_{4}^{4}+\varepsilon c\left|y\left(\theta_{t} \omega\right)\right|^{6}+c .
\end{aligned}
$$

For the last term of (45), by Lemma 13 again we get

$$
\begin{aligned}
- & \varepsilon \operatorname{Re}\left((\lambda+i \alpha(t)) \Delta z\left(\theta_{t} \omega\right), \Delta v\right) \\
& \leqslant \frac{\lambda}{4}\|\Delta v\|^{2}+\varepsilon c|\lambda+i \alpha(t)|^{2}\left\|\Delta z\left(\theta_{t} \omega\right)\right\|^{2} \\
& \leqslant \frac{\lambda}{4}\|\Delta v\|^{2}+\varepsilon c|\alpha(t)|^{2}\left\|\Delta z\left(\theta_{t} \omega\right)\right\|^{2}+\varepsilon c \lambda^{2}\left\|\Delta z\left(\theta_{t} \omega\right)\right\|^{2} \\
& \leqslant \frac{\lambda}{4}\|\Delta v\|^{2}+c|\alpha(t)|^{4}+\varepsilon c\left|y\left(\theta_{t} \omega\right)\right|^{6}+c,
\end{aligned}
$$

where $c=c(\lambda,\|\Delta h\|)$. Since, by (31) and Lemma 13 again,

$$
\begin{gathered}
\delta\|\nabla v\|^{2} \leqslant \frac{\lambda}{8}\|\Delta v\|^{2}+c\|v\|_{4}^{4}+c \\
-\operatorname{Re}(g(t), \Delta v) \leqslant \frac{4}{\lambda}\|g(x, t)\|^{2}+\frac{\lambda}{8}\|\Delta v\|^{2},
\end{gathered}
$$

from (45), (52), and (53) we conclude that

$$
\begin{aligned}
\frac{\mathrm{d}}{\mathrm{d} t}\|\nabla v\|^{2} \leqslant & c\left(\varepsilon\left|y\left(\theta_{t} \omega\right)\right|^{6}+1\right)\left(\|v\|_{4}^{4}+1\right) \\
& +c\left(\|g(x, t)\|^{2}+|\alpha(t)|^{4}\right),
\end{aligned}
$$

where $c$ is a positive constant independent of $\tau, \omega$, and $D$. Given $t \geqslant 0, \tau \in \mathbb{R}, \omega \in \Omega$, and $s \in] \tau-1, \tau$ [, integrating (55) over ]s, $\tau$ [ and by (20) we find that

$$
\begin{aligned}
\| \nabla v( & \left., \tau-t, \omega, v_{0}\right) \|^{2} \\
\leqslant & \left\|\nabla v\left(s, \tau-t, \omega, v_{0}\right)\right\|^{2} \\
& +c \int_{s}^{\tau}\left(\varepsilon\left|y\left(\theta_{\xi} \omega\right)\right|^{6}+1\right)\left(\|v(\xi)\|_{4}^{4}+1\right) \mathrm{d} \xi \\
& +c \int_{s}^{\tau}\left(\|g(x, \xi)\|^{2}+|\alpha(\xi)|^{4}\right) \mathrm{d} \xi \\
\leqslant & \left\|\nabla v\left(s, \tau-t, \omega, v_{0}\right)\right\|^{2} \\
& +c \int_{\tau-1}^{\tau}\left(\varepsilon r\left(\theta_{\xi} \omega\right)+1\right)\|v(\xi)\|_{4}^{4} \mathrm{~d} \xi \\
& +c \int_{\tau-1}^{\tau}\left(\varepsilon r\left(\theta_{\xi} \omega\right)+1\right) \mathrm{d} \xi \\
& +c \int_{\tau-1}^{\tau}\left(\|g(x, \xi)\|^{2}+|\alpha(\xi)|^{4}\right) \mathrm{d} \xi
\end{aligned}
$$

where $c$ is a positive constant independent of $\tau, \omega$, and $D$. Integrating (56) with respect to $s$ over ] $\tau-1, \tau$ [ and replacing $\omega$ with $\theta_{-\tau} \omega$, by (21) we derive that

$$
\begin{aligned}
& \left\|\nabla v\left(\tau, \tau-t, \theta_{-\tau} \omega, v_{0}\right)\right\|^{2} \\
& \leqslant \int_{\tau-1}^{\tau}\left\|\nabla v\left(\xi, \tau-t, \theta_{-\tau} \omega, v_{0}\right)\right\|^{2} \mathrm{~d} \xi \\
& \quad+(\varepsilon c r(\omega)+c) \int_{\tau-1}^{\tau}\left\|v\left(\xi, \tau-t, \theta_{-\tau} \omega, v_{0}\right)\right\|_{4}^{4} \mathrm{~d} \xi \\
& \quad+\operatorname{\varepsilon cr}(\omega)+c+c \int_{\tau-1}^{\tau}\left(\|g(x, \xi)\|^{2}+|\alpha(\xi)|^{4}\right) \mathrm{d} \xi
\end{aligned}
$$

where $c$ depends on $\gamma$ but is independent of $\varepsilon, \tau, \omega$, and $D$. Let $T=T(\tau, \omega, D) \geqslant 1$ be the same as in Lemma 17. Then from (57) and (42) it follows that

$$
\begin{aligned}
\left\|\nabla v\left(\tau, \tau-t, \theta_{-\tau} \omega, v_{0}\right)\right\|^{2} \\
\leqslant(\varepsilon c r(\omega)+c) e^{-\gamma \tau} \int_{-\infty}^{\tau} e^{\gamma s}\left(|\alpha(s)|^{4}+\|g(x, s)\|^{2}\right) \mathrm{d} s \\
\quad+(\varepsilon c r(\omega)+c)^{2}+c \int_{\tau-1}^{\tau}\left(\|g(x, \xi)\|^{2}+|\alpha(\xi)|^{4}\right) \mathrm{d} \xi \\
\leqslant(\varepsilon c r(\omega)+c) e^{-\gamma \tau} \int_{-\infty}^{\tau} e^{\gamma s}\left(|\alpha(s)|^{4}+\|g(x, s)\|^{2}\right) \mathrm{d} s \\
\quad+(\varepsilon c r(\omega)+c)^{2},
\end{aligned}
$$

which completes the proof.

To derive uniform estimates on the solutions $u$ of (13)(14), recall from (28) that

$$
u\left(\tau, \tau-t, \theta_{-\tau} \omega, u_{0}\right)=v\left(\tau, \tau-t, \theta_{-\tau} \omega, v_{0}\right)+z(\omega),
$$


where $v_{0}=u_{0}-z\left(\theta_{-t} \omega\right)$. Hence, for $X=H$ or $V$, we have

$$
\begin{aligned}
& \left\|u\left(\tau, \tau-t, \theta_{-\tau} \omega, u_{0}\right)\right\|_{X}^{2} \\
& \quad \leqslant 2\left\|v\left(\tau, \tau-t, \theta_{-\tau} \omega, v_{0}\right)\right\|_{X}^{2}+2\|z(\omega)\|_{X}^{2} \\
& \quad \leqslant 2\left\|v\left(\tau, \tau-t, \theta_{-\tau} \omega, v_{0}\right)\right\|_{X}^{2}+2\|h(x)\|_{X}^{2}(r(\omega)+1) .
\end{aligned}
$$

Moreover, by the temperance of $r(\omega)$ it is evident that $v_{0}$ comes from a nonautonomous random set in $\mathscr{D}_{\gamma}$ provided, and so does $u_{0}$. Therefore, Lemmas 16 and 18 imply the following lemma.

Lemma 19. Let assumptions of Lemma 12 hold. Then for every $\tau \in \mathbb{R}, \omega \in \Omega$, and $D \in \mathscr{D}_{\gamma}$, there exists a $T=$ $T(\tau, \omega, D) \geqslant 1$ and a positive constant $M$, which depends on $\gamma$ but is independent of $\varepsilon, \tau, \omega$, and $D$, such that the solution $u\left(t, \tau, \omega, u_{0}\right)$ with $u_{0} \in D\left(\tau-t, \theta_{-\tau} \omega\right)$ of (13)-(14) satisfies, for all $t \geqslant T$, that

$$
\begin{aligned}
& \left\|u\left(\tau, \tau-t, \theta_{-t} \omega, u_{0}\right)\right\|^{2} \\
& \leqslant M e^{-\gamma \tau} \int_{-\infty}^{\tau} e^{\gamma s}\left(|\alpha(t)|^{4}+\|g(x, s)\|^{2}\right) \mathrm{d} s \\
& \quad+\varepsilon M r(\omega)+M,
\end{aligned}
$$

and that

$$
\begin{aligned}
& \left\|u\left(\tau, \tau-t, \theta_{-\tau} \omega, u_{0}\right)\right\|_{V}^{2} \\
& \leqslant M(\varepsilon r(\omega)+1) e^{-\gamma \tau} \int_{-\infty}^{\tau} e^{\gamma s}\left(|\alpha(s)|^{4}+\|g\|^{2}\right) \mathrm{d} s \\
& \quad+M(\varepsilon r(\omega)+1)^{2}
\end{aligned}
$$

where $r(\omega)$ is the tempered random variable given by (20) and (21).

\section{Pullback Attractors for Stochastic G.-L. Equations}

4.1. Existence. In this part, for each $\varepsilon \in] 0,1]$ we establish the existence of the $\mathscr{D}_{\gamma}$-pullback attractor $\mathscr{A}_{\varepsilon}$ for system (13).

Consider the nonautonomous random set $E_{\varepsilon}=$ $\left\{E_{\varepsilon}(\tau, \omega)\right\}_{\tau, \omega}$ with

$$
E_{\varepsilon}(\tau, \omega):=\left\{u \in H:\|u\|_{V}^{2} \leqslant \mathscr{J}_{\varepsilon}(\tau, \omega)\right\}
$$

for each $\tau \in \mathbb{R}$ and $\omega \in \Omega$, where $\mathscr{F}_{\varepsilon}(\tau, \omega)$ is given by

$$
\begin{aligned}
\mathscr{J}_{\varepsilon}(\tau, \omega)= & M(\varepsilon r(\omega)+1) e^{-\gamma \tau} \int_{-\infty}^{\tau} e^{\gamma s}\left(|\alpha(s)|^{4}+\|g\|^{2}\right) \mathrm{d} s \\
& +M(\varepsilon r(\omega)+1)^{2},
\end{aligned}
$$

where $M$ is the constant found out by Lemma 19. It is evident that $\mathscr{J}_{\epsilon}(\tau, \cdot): \omega \rightarrow \mathbb{R}^{+}$is $(\mathscr{F}, \mathscr{B}(\mathbb{R}))$-measurable for each $\tau \in \mathbb{R}$. Moreover, by the temperance of $r(\omega)$ and assumption (iii) of Lemma 12 one can readily verify that

$$
\begin{aligned}
\lim _{t \rightarrow+\infty} e^{-\gamma t}\left\|E_{\epsilon}\left(\Theta_{-t} \tau, \theta_{-t} \omega\right)\right\|^{2} & =\lim _{t \rightarrow+\infty} e^{-\gamma t} \mathcal{J}_{\epsilon}\left(\Theta_{-t} \tau, \theta_{-t} \omega\right) \\
& =0
\end{aligned}
$$

which indicates by Definition 3 that $E_{\epsilon} \in \mathscr{D}_{\gamma}$. Therefore, by Definition 5, definition (28), and Lemma 19 (62), $E_{\epsilon}$ is a closed (and moreover, compact by Sobolev compactness embeddings) measurable $\mathscr{D}_{\gamma}$-pullback absorbing set for $\phi$. In addition, it is evident that $\phi$ is $\mathscr{D}_{\gamma}$-pullback asymptotically compact in $H$. Hence, by Lemma 10 we obtain the following existence result.

Theorem 20. Let assumptions of Lemma 12 hold. Then for each $\varepsilon \in] 0,1]$ the cocycle associated with problem (13)-(14) has a unique $\mathscr{D}_{\gamma}$-pullback attractor $\mathscr{A}_{\varepsilon}=\left\{A_{\varepsilon}(\tau, \omega)\right\}_{\tau, \omega}$ in $L^{2}(\mathscr{I})$.

4.2. Upper Semicontinuity. In the sequel we denote by $\phi_{\varepsilon}(t, \tau$, $\omega)$ the cocycle corresponding to the solution $u^{\varepsilon}\left(t, \tau, \omega, u_{0}^{\varepsilon}\right)$ with $u^{\varepsilon}(\tau)=u_{0}^{\varepsilon}$ of (13) (to indicate the dependence of $\varepsilon$ ), and let $\phi_{0}(t, \tau)$ be the solution operator generated by the deterministic nonautonomous system

$$
\frac{\mathrm{d} u}{\mathrm{~d} t}=(\lambda+i \alpha(t)) \Delta u-(\kappa+i \beta(t))|u|^{2} u+\delta u+g(x, t),
$$

$$
u(x, \tau)=u_{0}(x),\left.\quad u(x, \cdot)\right|_{\partial \mathscr{I}} \equiv 0,
$$

and given by

$$
\phi_{0}(t, \tau) u_{0}=u\left(t+\tau, \tau, u_{0}\right)
$$

where $u\left(t, \tau, u_{0}\right)$ with $u(\tau)=u_{0}$ solves the problem (66)(67). Analogously to cocycles $\phi_{\varepsilon}(t, \tau, \omega)$, under assumptions of Lemma $12 \phi_{0}(t, \tau)$ is a continuous operator from $H$ to $H$, and moreover, it has a $\mathscr{D}_{\gamma}$-pullback attractor $\mathscr{A}_{0}=\left\{A_{0}(\tau)\right\}_{\tau}$.

Lemma 21. Let assumptions of Lemma 12 hold. Then for every $T \in \mathbb{R}^{+}, \tau \in \mathbb{R}, \omega \in \Omega$, and $u_{0}^{\varepsilon}, u_{0} \in X$ with $u_{0}^{\varepsilon} \rightarrow u_{0}$ as $\varepsilon \downarrow 0$, it holds that

$$
\lim _{\varepsilon \downarrow 0} \phi_{\varepsilon}(T, \tau, \omega) u_{0}^{\varepsilon}=\phi_{0}(T, \tau) u_{0} .
$$

Proof. Let $v_{\varepsilon}\left(t, \tau, \omega, v_{0}^{\varepsilon}\right)=u_{\varepsilon}\left(t, \tau, \omega, u_{0}^{\varepsilon}\right)-\varepsilon z\left(\theta_{t} \omega\right)$ with $v_{0}^{\varepsilon}(\omega)=u_{0}^{\varepsilon}-\varepsilon z(\omega)$ and write $W(t, \tau, \omega)=v_{\varepsilon}\left(t, \tau, \omega, v_{0}^{\varepsilon}\right)-$ $u\left(t, \tau, u_{0}\right)$. Then by (23) and (66), $W$ satisfies

$$
\begin{aligned}
\frac{\mathrm{d} W}{\mathrm{~d} t}= & (\lambda+i \alpha(t)) \Delta W-(\kappa+i \beta(t))\left(f\left(v+\varepsilon z\left(\theta_{t} \omega\right)\right)-f(u)\right) \\
& +\delta W+\varepsilon(\lambda+i \alpha(t)) \Delta z\left(\theta_{t} \omega\right)
\end{aligned}
$$

where $f(s)=|s|^{2} s$. Since, for each fixed $\omega \in \Omega$,

$$
\|W(\tau)\|=\left\|v_{0}^{\varepsilon}-u_{0}\right\|=\left\|u_{0}^{\varepsilon}-\varepsilon z(\omega)-u_{0}\right\| \longrightarrow 0 \quad \text { as } \varepsilon \downarrow 0,
$$


there exists a $\left.\left.\varepsilon_{0} \in\right] 0,1\right]$ such that, for the fixed $\omega$,

$$
\left.\left.\left\|v_{0}^{\varepsilon}\right\|^{2} \leqslant\left\|u_{0}\right\|^{2}+1 \quad \forall \varepsilon \in\right] 0, \varepsilon_{0}\right] .
$$

Taking the inner product of (70) with $W$ in $H$ and taking the real part we have

$$
\begin{aligned}
\frac{1}{2} \frac{\mathrm{d}}{\mathrm{d} t}\|W\|^{2}+\lambda\|\nabla W\|^{2} \\
=\delta\|W\|^{2}-\operatorname{Re}\left((\kappa+i \beta(t))\left(f\left(u_{\varepsilon}\right)-f(u)\right), W\right) \\
\quad+\varepsilon \operatorname{Re}\left((\lambda+i \alpha(t)) \Delta z\left(\theta_{t} \omega\right), W\right) .
\end{aligned}
$$

In the sequel we consider $\left.\varepsilon \in] 0, \varepsilon_{0}\right]$. By the condition $\beta(t) \leqslant$ $\sqrt{3} \kappa$, for all $t \in \mathbb{R}$, we have

$$
\begin{aligned}
& -\operatorname{Re}\left((\kappa+i \beta(t))\left(f\left(u_{\varepsilon}\right)-f(u)\right), W\right) \\
& =-\operatorname{Re}\left((\kappa+i \beta(t))\left(\left|u_{\varepsilon}\right|^{2}\left(u_{\varepsilon}-u\right)+\left(\left|u_{\varepsilon}\right|^{2}-|u|^{2}\right) u\right), W\right) \\
& \leqslant 2 \kappa \int_{\mathscr{I}}\left(\left|u_{\varepsilon}\right|^{2}\left|W+\varepsilon z\left(\theta_{t} \omega\right)\right|+\left|u_{\varepsilon}+u\left\|W+\varepsilon z\left(\theta_{t} \omega\right)\right\| u\right|\right) \\
& \quad \times|W| \mathrm{d} x \\
& \leqslant c\left\|u_{\varepsilon}\right\|_{\infty}^{2}\left(\varepsilon\left\|z\left(\theta_{t} \omega\right)\right\|^{2}+\|W\|^{2}\right) \\
& +c\left(\left\|u_{\varepsilon}\right\|_{\infty}^{2}+\|u\|_{\infty}^{2}\right)\left(\varepsilon\left\|z\left(\theta_{t} \omega\right)\right\|^{2}+\|W\|^{2}\right) \\
& \leqslant c\left(\left\|u_{\varepsilon}\right\|_{\infty}^{2}+\|u\|_{\infty}^{2}\right)\|W\|^{2}+\varepsilon c\left\|z\left(\theta_{t} \omega\right)\right\|^{2} \\
& \quad \times\left(\left\|u_{\varepsilon}\right\|_{\infty}^{2}+\|u\|_{\infty}^{2}\right),
\end{aligned}
$$

where $c$ depends on $\kappa$ but is independent of $\varepsilon$. Similarly by Lemma 13 we have

$$
\begin{aligned}
& \varepsilon \operatorname{Re}\left((\lambda+i \alpha(t)) \Delta z\left(\theta_{t} \omega\right), W\right) \\
& \quad \leqslant \varepsilon c\left\|\Delta z\left(\theta_{t} \omega\right)\right\|^{2}\left(1+|\alpha(t)|^{2}\right)+\varepsilon\|W\|^{2} .
\end{aligned}
$$

Then from (73)-(75) it follows that

$$
\begin{aligned}
& \frac{\mathrm{d}}{\mathrm{d} t}\|W(t, \tau, \omega)\|^{2} \\
& \leqslant c\left(\left\|u_{\varepsilon}\left(t, \tau, \omega, u_{0}^{\varepsilon}\right)\right\|_{\infty}^{2}+\|u\|_{\infty}^{2}+1\right)\|W(t, \tau, \omega)\|^{2} \\
& \quad+\varepsilon c\left\|z\left(\theta_{t} \omega\right)\right\|^{2}\left(\left\|u_{\varepsilon}\left(t, \tau, \omega, u_{0}^{\varepsilon}\right)\right\|_{\infty}^{2}+\|u\|_{\infty}^{2}+|\alpha(t)|^{2}+1\right) \\
& \leqslant c N(t, \tau, \omega)\|W(t, \tau, \omega)\|^{2}+\varepsilon c\left\|z\left(\theta_{t} \omega\right)\right\|^{2} N(t, \tau, \omega),
\end{aligned}
$$

where $c$ is a positive constant independent of $\varepsilon$ and $N(t, \tau, \omega)$ is given by

$$
N(t, \tau, \omega):=\sup _{0<0 \leqslant \varepsilon_{0}}\left\|u_{\varepsilon}\left(t, \tau, \omega, u_{0}^{\varepsilon}\right)\right\|_{\infty}^{2}+\|u\|_{\infty}^{2}+|\alpha(t)|^{2}+1 .
$$

Let $T>\tau$ be an arbitrarily fixed time. By Agmon inequality (49) and (62) we have

$$
\int_{\tau}^{\tau+T} N\left(s, \tau, \theta_{-T} \omega\right) \mathrm{d} s<+\infty .
$$

Therefore, replacing $\omega$ with $\theta_{-T} \omega$ in (76) and by Gronwall lemma we have

$$
\begin{aligned}
& \left\|W\left(\tau+T, \tau, \theta_{-T} \omega\right)\right\|^{2} \\
& \leqslant e^{c \int_{\tau}^{\tau+T} N\left(s, \tau, \theta_{-T} \omega\right) \mathrm{d} s}\|W(\tau)\|^{2} \\
& +\varepsilon \mathcal{C} \int_{\tau}^{\tau+T} e^{\int_{s}^{\tau+T} N\left(v, \tau, \theta_{-T} \omega\right) \mathrm{d} v}\left\|z\left(\theta_{s-T} \omega\right)\right\|^{2} N\left(s, \tau, \theta_{-T} \omega\right) \mathrm{d} s \\
& \leqslant e^{c \int_{\tau}^{\tau+T} N\left(s, \tau, \theta_{-T} \omega\right) \mathrm{d} s}\|W(\tau)\|^{2} \\
& +\varepsilon \mathcal{E c}\left(\sup _{\tau-T<s<\tau}\left|y\left(\theta_{s} \omega\right)\right|^{2}\right) e^{\int_{\tau}^{\tau+T} N\left(v, \tau, \theta_{-T} \omega\right) \mathrm{d} v} \\
& \cdot \int_{\tau}^{\tau+T} N\left(s, \tau, \theta_{-T} \omega\right) \mathrm{d} s
\end{aligned}
$$

Since $W(t, \tau, \omega)=u_{\varepsilon}\left(t, \tau, \omega, u_{0}^{\varepsilon}\right)-\varepsilon z\left(\theta_{t} \omega\right)-u\left(t, \tau, u_{0}\right)$, from (71) and (79) it follows that

$$
\begin{aligned}
&\left\|u_{\varepsilon}\left(\tau+T, \tau, \theta_{-T} \omega, u_{0}^{\varepsilon}\right)-u\left(\tau+T, \tau, u_{0}\right)\right\|^{2} \\
& \leqslant 2\left\|W\left(\tau+T, \tau, \theta_{-T} \omega\right)\right\|^{2}+2 \varepsilon^{2}\left\|z\left(\theta_{\tau} \omega\right)\right\|^{2} \longrightarrow 0, \\
& \text { as } \varepsilon \downarrow 0,
\end{aligned}
$$

which completes the proof.

Theorem 22. Let assumptions of Lemma 12 hold. Then for every $\tau \in \mathbb{R}$ and $\omega \in \Omega$,

$$
\lim _{\varepsilon \downarrow 0} \operatorname{dist}_{H}\left(A_{\varepsilon}(\tau, \omega), A_{0}(\tau)\right)=0 .
$$

Proof. We prove that the result verifies the four conditions of Lemma 11. First, it is evident that condition (i) is actually proved by Lemma 21 . To verify the rest, let

$$
\begin{aligned}
R_{0}(\tau) & =\left.\mathscr{J}_{\varepsilon}(\tau, \omega)\right|_{\varepsilon=0} \\
& =M e^{-\gamma \tau} \int_{-\infty}^{\tau} e^{\gamma s}\left(|\alpha(s)|^{4}+\|g\|^{2}\right) \mathrm{d} s+M,
\end{aligned}
$$

where $M$ is the constant as in (63). Since $E_{\varepsilon}=\left\{E_{\varepsilon}(\tau, \omega)\right\}_{\tau, \omega}$ for each $\varepsilon \in] 0,1]$ defined by (63) is a compact $\mathscr{D}_{\gamma}$-pullback absorbing set for $\phi_{\varepsilon}$ in $H$, conditions (ii) and (iii) of Lemma 11 hold and condition (iv) follows from the fact that

$$
\bigcup_{\varepsilon \in] 0,1]} A_{\varepsilon}(\tau, \omega) \subseteq \bigcup_{\varepsilon \in] 0,1]} E_{\varepsilon}(\tau, \omega) \subseteq E_{1}(\tau, \omega) .
$$

Then the theorem is concluded.

\section{Conflict of Interests}

The authors declare that there is no conflict of interests regarding the publication of this paper. 


\section{Acknowledgments}

The authors would like to express their thanks to anonymous reviewers for their valuable comments. This work was partially supported by the NSFC Grants 11071199 and 11371183.

\section{References}

[1] R. Temam, Infinite Dimensional Dynamical Systems in Mechanics and Physics, Springer, New York, NY, USA, 2nd edition, 1997.

[2] M. I. Vishik and V. V. Chepyzhov, "Non-autonomous GinzburgLandau equation and its at tractors," Sbornik: Mathematics, vol. 196, no. 6, pp. 791-815, 2005.

[3] Y. Li and B. Guo, "Global existence of solutions to the derivative 2D Ginzburg-Landau equation," Journal of Mathematical Analysis and Applications, vol. 249, no. 2, pp. 412-432, 2000.

[4] H. Gao and J. Duan, "On the initial-value problem for the generalized two-dimensional Ginzburg-Landau equation," Journal of Mathematical Analysis and Applications, vol. 216, no. 2, pp. 536-548, 1997.

[5] P. Clement, N. Okazawa, M. Sobajima, and T. Yokota, "A simple approach to the Cauchy problem for complex Ginzburg-Landau equations by compactness methods," Journal of Differential Equations, vol. 253, no. 4, pp. 1250-1263, 2012.

[6] D. Yang, "The asymptotic behavior of the stochastic GinzburgLandau equation with multiplicative noise," Journal of Mathematical Physics, vol. 45, no. 11, pp. 4064-4076, 2004.

[7] G. Wang, B. Guo, and Y. Li, "The asymptotic behavior of the stochastic Ginzburg-Landau equation with additive noise," Applied Mathematics and Computation, vol. 198, no. 2, pp. 849857, 2008.

[8] B. Wang, "Sufficient and necessary criteria for existence of pullback attractors for non-compact random dynamical systems," Journal of Differential Equations, vol. 253, no. 5, pp. 1544-1583, 2012.

[9] B. Wang, "Existence and upper semicontinuity of attractors for stochastic equations with deterministic non-autonomous terms," Stochastics and Dynamics, 2014.

[10] B. Wang, "Random attractors for non-autonomous stochastic wave equations with multiplicative noise," Discrete and Continuous Dynamical Systems A, vol. 34, no. 1, pp. 269-300, 2014.

[11] B. Wang, "Asymptotic behavior of stochastic wave equations with critical exponents on $R_{3}$," Transactions of the American Mathematical Society, vol. 363, no. 7, pp. 3639-3663, 2011.

[12] L. Arnold, Random Dynamical Systems, Springer, Berlin, Germany, 1998.

[13] H. Crauel, A. Debussche, and F. Flandoli, "Random attractors," Journal of Dynamics and Differential Equations, vol. 9, no. 2, pp. 307-341, 1997.

[14] B. Wang, "Upper semicontinuity of random attractors for noncompact random dynamical systems," Electronic Journal of Differential Equations, vol. 2009, article 139, 18 pages, 2009.

[15] W. Zhao and Y. Li, " $\left(\mathrm{L}^{2}, \mathrm{~L}^{p}\right)$-random attractors for stochastic reaction-diffusion equation on unbounded domains," Nonlinear Analysis: Theory, Methods \& Applications, vol. 75, no. 2, pp. 485-502, 2012.

[16] M. Yang and P. E. Kloeden, "Random attractors for stochastic semi-linear degenerate parabolic equations," Nonlinear Analysis: Real World Applications, vol. 12, no. 5, pp. 2811-2821, 2011.

[17] V. V. Chepyzhov and M. I. Vishik, Attractors for Equations of Mathematical Physics, vol. 49, American Mathematical Society, Providence, RI, USA, 2002. 


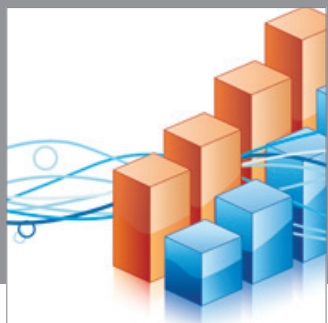

Advances in

Operations Research

mansans

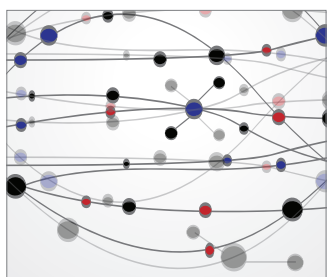

The Scientific World Journal
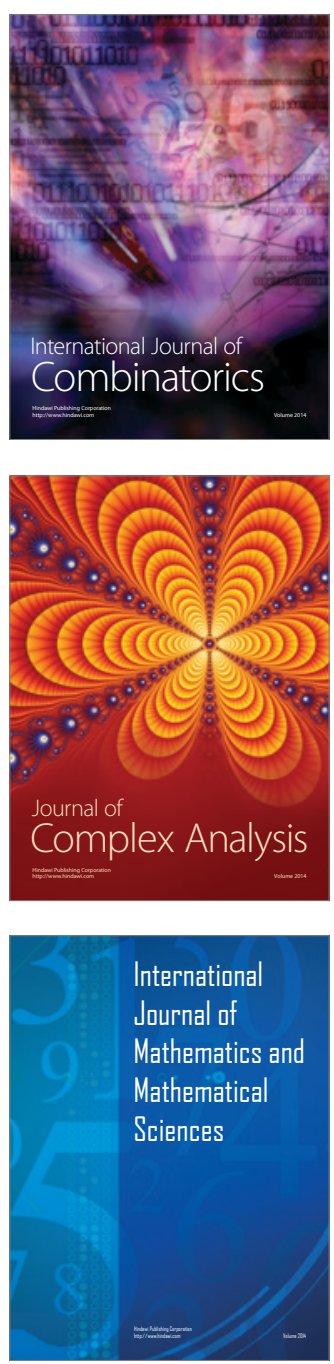
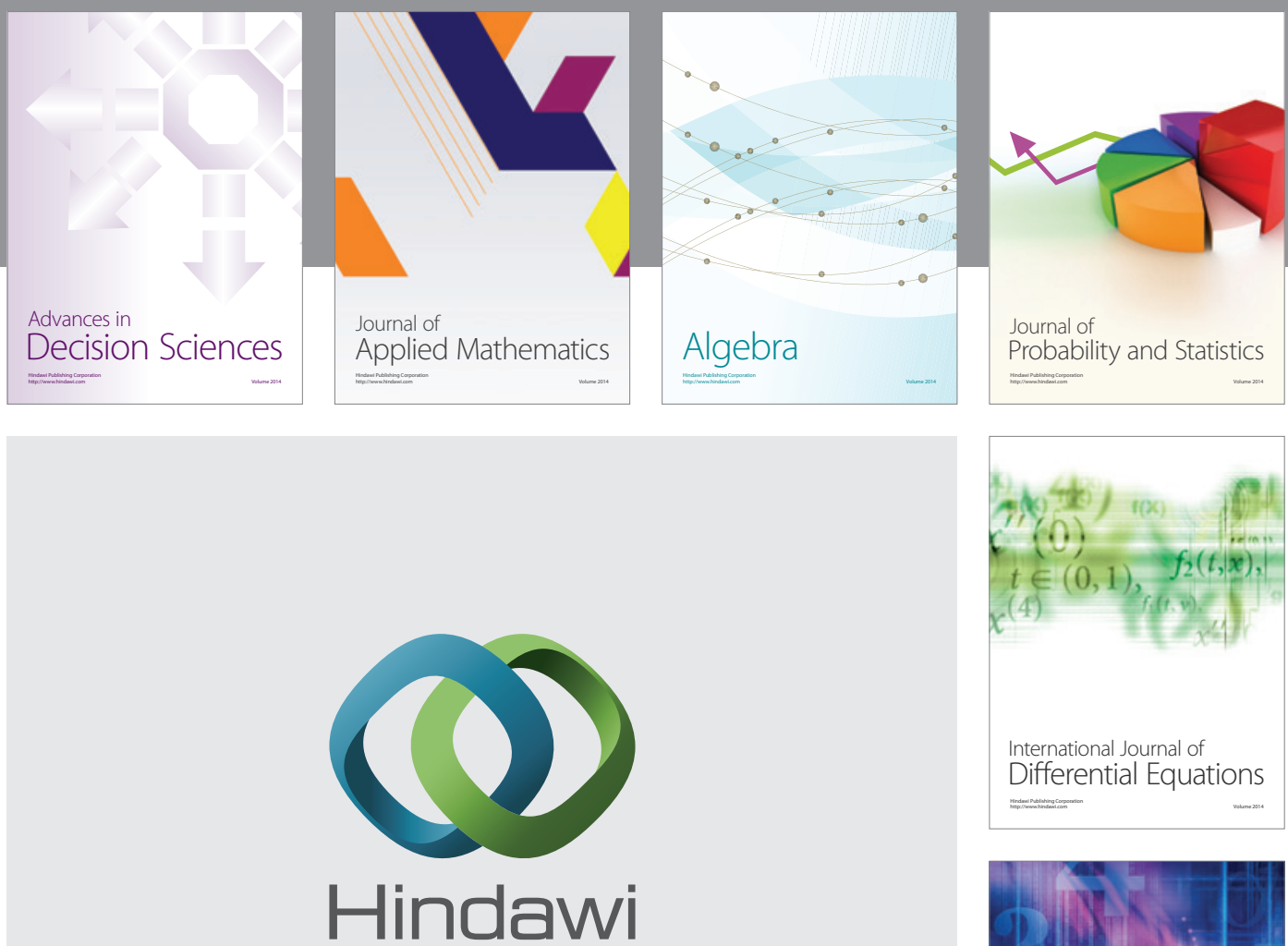

Submit your manuscripts at http://www.hindawi.com
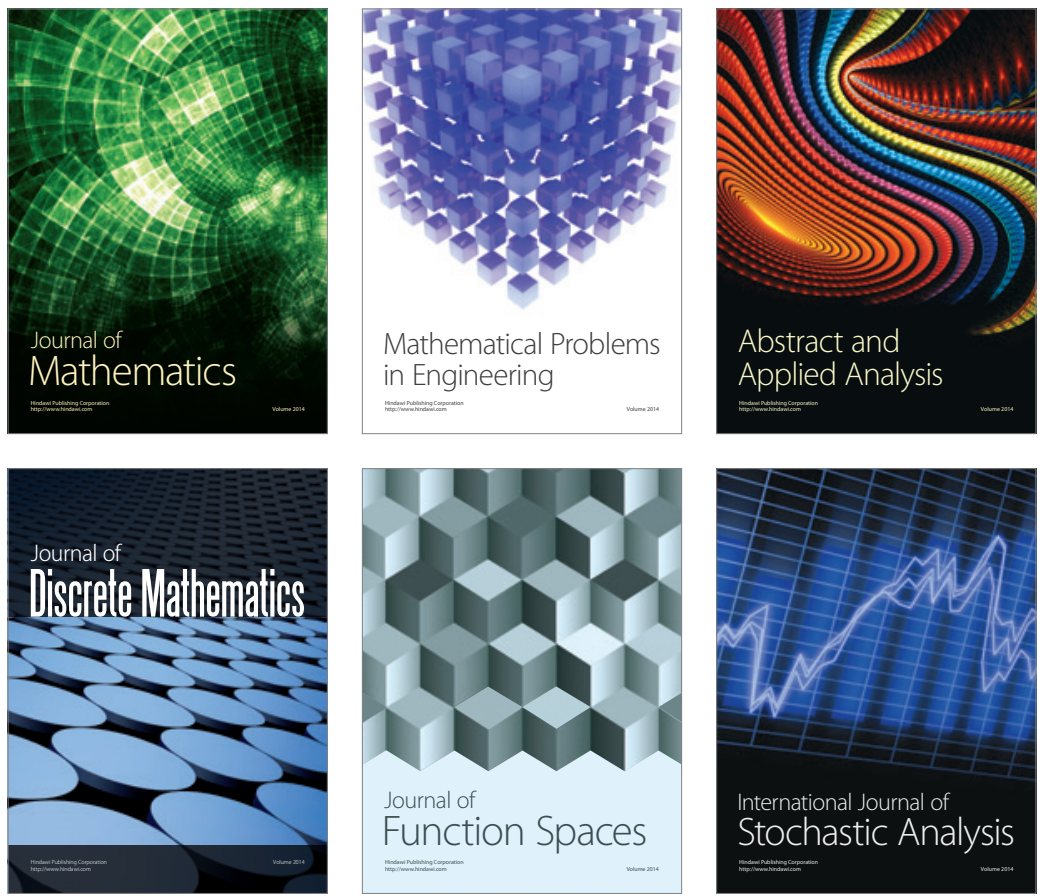

Journal of

Function Spaces

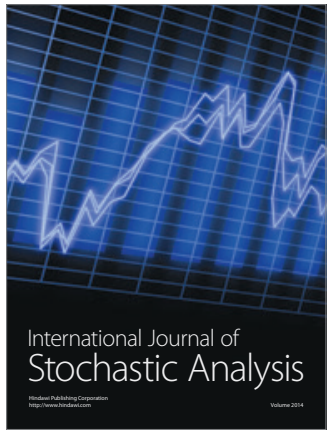

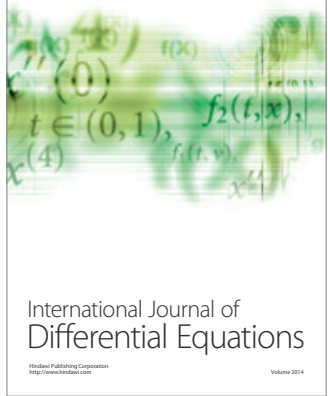
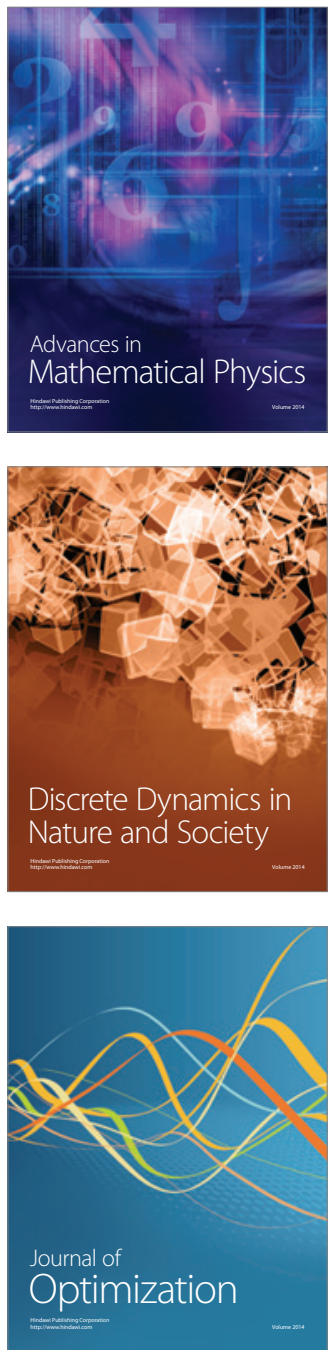